\title{
Diagnosis and management of hyalinizing trabecular tumor of the thyroid: a single-institution experience
}

\author{
Yasuhiro Ito ${ }^{1)}$, Mitsuyoshi Hirokawa ${ }^{2)}$, Kazuyoshi Kousaka ${ }^{3)}$, Mitsuru Ito ${ }^{3)}$, Minoru Kihara' ${ }^{1)}$ \\ Akihiro Miya ${ }^{1)}$ and Akira Miyauchi ${ }^{1)}$ \\ 1) Departments of Surgery, Kuma Hospital, Kobe, Hyogo 650-0011, Japan \\ 2) Departments of Diagnostic Pathology, Kuma Hospital, Kobe, Hyogo 650-0011, Japan \\ 3) Departments of Internal Medicine, Kuma Hospital, Kobe, Hyogo 650-0011, Japan
}

\begin{abstract}
Hyalinizing trabecular tumor (HTT) of the thyroid is a mostly benign disease. Its cytological and pathological diagnosis is often difficult, because HTT cells and papillary thyroid carcinoma (PTC) cells share similar features (e.g., intranuclear cytoplasmic inclusions and nuclear grooves). At our institution, 38 patients were diagnosed as or highly suspected of having HTT without the possibility of PTC, based on cytology: 19 of these patients underwent immediate surgery (surgery group) and the remaining 19 underwent active surveillance without surgery (AS group). The surgery-group patients' tumor sizes were significantly larger $(p<0.0001)$ than those in the AS group. During AS (median 38 months), only one patient $(5 \%)$ showed tumor enlargement by $\geq 3 \mathrm{~mm}$; the AS was continued. Of the 34 patients pathologically diagnosed with HTT, 22 $(65 \%)$ were cytologically diagnosed or highly suspected as having HTT without the possibility of PTC. Of the nine patients who were suspected to have HTT but PTC was possible and surgery was performed, two (22\%) and seven (78\%) were pathologically diagnosed as having PTC and HTT, respectively. Five patients were cytologically diagnosed with PTC, but pathologically diagnosed as having HTT. No patients showed HTT recurrence during postoperative follow-up (median 60 months). These findings suggest that (1) active surveillance can be a valid strategy for managing tumors that are cytologically diagnosed as HTT with no possibility of PTC; (2) surgery is recommended for tumors suspected of being HTT but may be PTC, and (3) the prognosis of HTT in both the AS and surgery groups was excellent.
\end{abstract}

Key words: Hyalinizing trabecular tumor, Thyroid, Diagnosis, Treatments

HYALINIZING TRABECULAR TUMOR (HTT) of the thyroid was proposed by Carney et al. in 1987 as a subtype of follicular adenoma [1]. However, the cytopathological findings of HTT are similar to those of papillary thyroid carcinoma (PTC); e.g., intranuclear cytoplasmic inclusions and nuclear grooves [2-4]. HTT and PTC have their own cytological features: e.g., spindled and elongated cell shape, yellow body in the cytoplasm, and hyaline material in the background for HTT; for PTC, e.g., round or oval cell shape, septate vacuoles in the cytoplasm, and psammoma body in the background $[5,6]$. Nevertheless, it is often difficult to reach a differential diagnosis based on cytology and pathology from PTC. In 1995, Hirokawa et al. reported that the staining of MIB-1 (a monoclonal antibody of Ki-67,

Submitted Feb. 24, 2021; Accepted Jun. 9, 2021 as EJ21-0143 Released online in J-STAGE as advance publication Jul. 6, 2021 Correspondence to: Yasuhiro Ito, Department of Surgery, Kuma Hospital, 8-2-35, Shimoyamate-dori, Chuo-ku, Kobe, Hyogo 650-0011, Japan.

E-mail: ito01@kuma-h.or.jp which normally reacts in cell nuclei in the late G1, S, G2, and $\mathrm{M}$ phases) was detected in the cell membrane of HTT cells $[7,8]$, and this finding can be helpful to diagnose HTT.

Carney et al. studied the pathological specimens and clinical outcomes of 116 patients with tumors of this pathologic subtype, and in 2008 they reported that one tumor had vascular invasion, capsular penetration, and pulmonary metastasis, while the others had no malignant findings on pathology and clinical courses [9]. They thus concluded that HTTs are "almost all benign" [9]. To date, however, no clinical or pathological studies conducted by a single institution have been published about HTT. From 2007, one of the coauthors (M.H.) has actively diagnosed HTT based on both cytology and pathology. Although our hospital did not have an established management strategy, we recommended immediate surgery or active surveillance for tumors that were cytologically diagnosed as or highly suspected of being HTT with no possibility of being PTC, based on the discretion of attending physicians. In contrast, we have generally 
performed surgery for tumors that are suspected of being HTT but also present the possibility of being PTC based on cytology. In the present report, we describe our single-institution experience with the diagnosis and management of HTTs in order to elucidate some clinical questions, such as how HTT can be accurately diagnosed based on cytology, whether or not an HTT grows when managed by active surveillance, and what the prognoses of patients with HTT are after surgery.

\section{Patients and Methods}

Between 2007 and 2019, 51 patients were diagnosed as or suspected of having HTT based on cytology at our institution (Kuma Hospital, Kobe, Japan). During the same period, 46 patients were pathologically diagnosed as having HTT; 12 of these HTTs were incidentally detected on a pathological examination, and these patients were deleted from the patient series of this study. All cytological and pathological specimens were examined by one thyroid pathologist (M.H.) who is a coauthor of this study. The cytological and pathological diagnoses were made based on the typical features for HTT as described [5].

MIB-1 immunostaining was routinely performed for the pathological specimens to confirm the diagnoses of HTT. However, MIB-1 immunostaining for cytological specimens was performed only when HTT was suspected but its typical cytological features could not be adequately detected. The details of the MIB-1 immunostaining technique were as described [10]. We used an antiKi-67 antibody (MIB-1, Dako, Glostrup, Denmark) (dilution 1:200 for immunocytochemistry and 1:400 for immunohistochemistry), and the antigen retrieval was carried out manually using heat-induced epitope retrieval (98 $\left.{ }^{\circ} \mathrm{C}, 60 \mathrm{~min}\right)$.

An ultrasound (US) examination was performed for all patients except for the 12 patients whose HTTs were incidentally detected by a pathological examination. These nodules were scored according to our own US classification system established by Yokozawa and Miyauchi et al. [11]. Intranodular and peripheral blood flows were evaluated by attending technicians and classified into negative, $1+, 2+$, and $3+$ based on the richness of the flow. If a patient underwent a US examination twice or more, we used the data with the highest flow score.

For the patients who underwent active surveillance, an evaluation of the tumor (mainly its size and shape) by US was conducted once or twice per year. For the patients who underwent surgery, we asked them to visit our hospital at least once a year for the determination of the presence/absence of new abnormal findings on US.
The Mann-Whitney $U$-test and the Chi-square test were used for the statistical analyses with the software StatFlex (Artec, Osaka, Japan). A $p$-value $<0.05$ was regarded as significant. The study was approved by the ethical committee at Kuma Hospital (No. 20200709-1).

\section{Results}

\section{The series of patients who were diagnosed as or suspected of having HTT based on cytology}

We divided the 51 patients who were diagnosed as or suspected of having HTT based on cytology into two categories based on the cytological findings. The first category was the tumors diagnosed as or highly suspected of being HTT without the possibility of PTC ( $n=$ 41 ), and the other category was the tumors that were suspected of being or were possible HTT on cytology and diagnosed as HTT based on pathology $(n=10)$. We recommended active surveillance or surgery for these patients based on the discretion of their attending physicians. Nineteen patients underwent active surveillance (the AS group) and 19 underwent immediate surgery (the surgery group). The surgery group included three patients who underwent observation before the cytological examination as they had benign nodules (passive surveillance) for 29, 67, and 294 months, respectively, but after the cytological diagnosis as HTT, immediate surgery was performed based on their physicians' recommendations. For the remaining three of the 41 patients, we had to recommend surgery for coexisting thyroid diseases (PTC with clinical node metastasis for two patients and Graves' disease with large goiter for one patient). All 22 patients $(19+3)$ who underwent surgery were also diagnosed as having HTT based on a postoperative pathology examination.

Another category was the tumors suspected of being or were possible HTTs with the possibility of PTC $(n=$ 10). Since PTC could not be denied, we recommended surgery for nine of these 10 patients. Seven (78\%) and two $(22 \%)$ patients' tumors were pathologically diagnosed as HTT and PTC, respectively. The remaining patient had a tumor measuring $5 \mathrm{~mm}$ and was observed because the attending physician suspected that the tumor was papillary microcarcinoma. Fig. 1 is the flow chart of the diagnosis and treatment of the cases in the present patient series.

Table 1 summarizes the relationships between clinical factors and the two patient groups (surgery and AS). The tumor size was significantly larger in the surgery group compared to the AS group ( $p<0.0001)$. Other factors such as patient age, gender, US class, and intranodular and peripheral blood flows did not differ significantly between the two groups. 


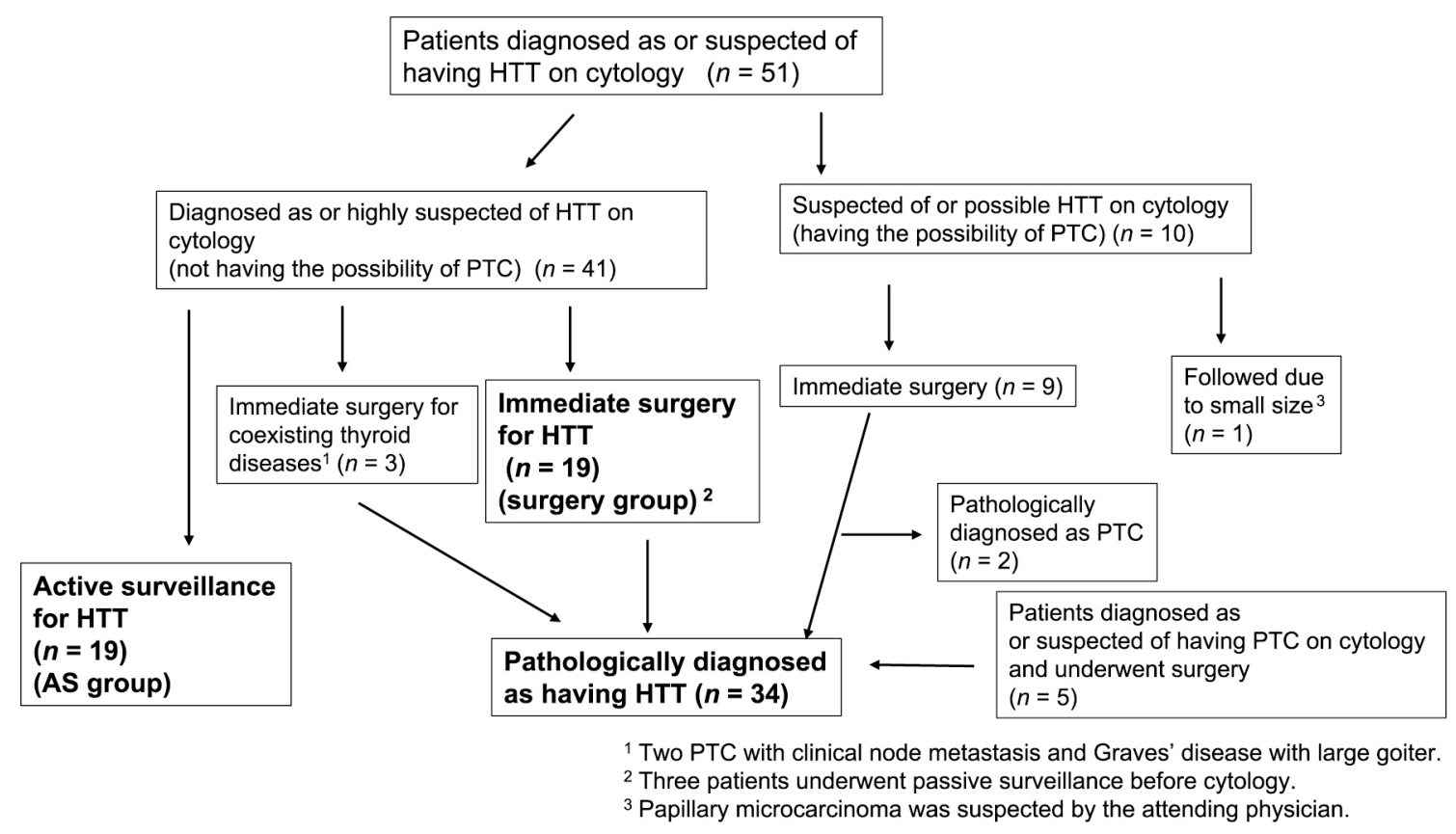

Fig. 1 A flow chart of the diagnosis and treatment of hyalinizing trabecular tumor (HTT) in our series.

Table 1 Backgrounds and clinical features of the 38 patients who were cytologically diagnosed as or highly suspected of having HTT*

\begin{tabular}{|c|c|c|c|c|}
\hline Variable & $\begin{array}{l}\text { AS group } \\
(n=19)\end{array}$ & $\begin{array}{l}\text { Surgery group }{ }^{1} \\
\quad(n=19)\end{array}$ & $\begin{array}{c}\text { No. of patients } \\
(\%)\end{array}$ & $p$-value \\
\hline Median age, yrs & $59(40-77)$ & $59(38-75)$ & & 0.9650 \\
\hline Gender: Male & $4(21 \%)$ & $1(5 \%)$ & $5(13 \%)$ & \multirow{2}{*}{0.1500} \\
\hline Female & $15(79 \%)$ & $18(95 \%)$ & $33(87 \%)$ & \\
\hline Median tumor size, $\mathrm{mm}$ & $14(5-29)$ & $26(15-60)$ & 38 & $<0.0001$ \\
\hline \multicolumn{5}{|l|}{ US class: } \\
\hline 2 & $1(5 \%)$ & 0 & $1(3 \%)$ & \multirow{4}{*}{0.3870} \\
\hline 2.5 & $1(5 \%)$ & $1(5 \%)$ & $2(6 \%)$ & \\
\hline 3 & $17(90 \%)$ & $16(85 \%)$ & $33(85 \%)$ & \\
\hline 3.5 & 0 & $2(10 \%)$ & $2(6 \%)$ & \\
\hline \multicolumn{5}{|l|}{ Intranodular blood flow: } \\
\hline $1+$ & $3(16 \%)$ & $7(37 \%)$ & $10(26 \%)$ & \multirow{3}{*}{0.3377} \\
\hline $2+$ & $12(63 \%)$ & $9(47 \%)$ & $21(56 \%)$ & \\
\hline $3+$ & $4(21 \%)$ & $3(16 \%)$ & $7(18 \%)$ & \\
\hline \multicolumn{5}{|l|}{ Peripheral blood flow: } \\
\hline $1+$ & $3(16 \%)$ & $5(26 \%)$ & $8(21 \%)$ & \multirow{3}{*}{0.6878} \\
\hline $2+$ & $9(48 \%)$ & $9(48 \%)$ & $18(47 \%)$ & \\
\hline $3+$ & $7(36 \%)$ & $5(26 \%)$ & $12(32 \%)$ & \\
\hline \multicolumn{5}{|c|}{$\begin{array}{l}\text { MIB-1 immunostaining of cytological } \\
\text { specimens: }\end{array}$} \\
\hline Positive & $7(100 \%)$ & $4(80 \%)$ & $11(92 \%)$ & \multirow[t]{3}{*}{0.4167} \\
\hline Negative & 0 & $1(20 \%)$ & $1(8 \%)$ & \\
\hline Not done & 12 & 14 & 26 & \\
\hline
\end{tabular}

* Three patients who underwent surgery for other thyroid diseases were excluded.

${ }^{1}$ The surgery group include three patients who underwent observation (passive surveillance) before cytological diagnosis and underwent surgery immediately after cytology. All 19 cases in the surgery group were pathologically diagnosed as having HTT. AS, active surveillance; HTT, hyalinizing trabecular tumor; US, ultrasound. 
The follow-up observation period of the 19 patients in the AS group ranged from 9 to 147 months (median 38 months). Only one patient (5\%) showed tumor enlargement by $\geq 3 \mathrm{~mm}$ (maximal dia.) after 28 months; the tumors of the remaining 18 patients showed no change in size. None of the patients, including the single enlargedtumor case, underwent conversion surgery during the observation period.

MIB-1 immunostaining of cytological specimens was performed for 12 of 41 patients (32\%) (seven in the AS group and five in the surgery group), and cell membranous staining for MIB-1 was detected in 11 patients; the remaining patient's tumor (measuring $39 \mathrm{~mm}$ ) was negative for MIB-1 staining, and this patient underwent surgery (Table 1). Of the 7 patients suspected of having HTT or with possible HTT, four underwent MIB-1 immunostaining on cytological specimens, and all were judged as negative (Table 2).

\section{The series of patients who were diagnosed as having HTT based on a pathological examination}

In our series, 34 patients were pathologically diagnosed as HTT on pathological examination. Of these, 22 $(65 \%)(19+3$ patients) were diagnosed as or highly suspected of having HTT on cytology, and seven (21\%) (92 patients) were suspected of having or with possible HTT, but the possibility of PTC could not be denied on cytology. Five patients (14\%) were diagnosed as or suspected of having PTC on cytology but were diagnosed as having HTT based on pathology (Fig. 1). Table 2 summarizes the backgrounds and clinicopathological features of the 34 patients diagnosed with HTT based on a pathological examination.

MIB-1 staining was performed for cytology specimens in only 11 patients. Five patients (45\%) showed MIB-1 membranous staining, but the remaining six (55\%) did not (Table 2). In contrast, all 34 of the above-described cases underwent MIB-1 immunostaining for pathological specimens for a confirmation of the diagnosis, and membranous MIB-1 immunostaining was detected in all of them. Four patients underwent a total thyroidectomy because of coexisting thyroid disease or a large tumor size. One patient underwent a modified radical neck dissection because of the coexistence of PTC with clinical lateral node metastasis. To date, none of these patients has shown recurrence of HTT (follow-up period, 1-151 months; median 60 months).

\section{Discussion}

The results of this study demonstrated that (1) the tumor sizes of the patients in AS group were significantly smaller than those of the patients in the surgery group, and no other features significantly differed between the two groups; (2) of the 19 patients who underwent active surveillance, only one (5\%) showed tumor enlargement $\geq 3 \mathrm{~mm}$; (3) of the 34 patients who were diagnosed as having HTT based on a pathological examination, HTT was diagnosed or highly suspected (without the possibility of PTC) in 22 (65\%) on cytology, and (4) none of the patients who underwent surgery showed the recurrence of HTT during postoperative follow-up.

At our hospital, we recommended immediate surgery for patients suspected of having or with possible HTT but whose possibility of PTC could not be denied on cytology. Indeed, as shown in Fig. 1, two of the nine patients $(22 \%)$ in this category were pathologically diagnosed as having PTC, indicating that the recommendation of surgery for such patients is considered appropriate. In contrast, for patients with tumors that are diagnosed as or highly suspected of being HTT but without the possibility of being PTC, we have recommended surgery or active surveillance based on the attending physicians' discretion. Interestingly, the tumor size was significantly smaller in the AS group compared to the surgery group, indicating that the tumor size is an important factor for physicians when selecting the management strategy for HTT, rather than US findings including intranodular and marginal blood flows.

In the series described by Carney et al. [9], one patient showed an aggressive behavior, i.e., vascular invasion, capsular penetration, and pulmonary metastasis, and these aggressive signs should be detected on imaging studies such as ultrasound and CT scan at the time of diagnosis. In our present series, none of the patients with HTT showed such aggressive signs, and the outcomes of the patients in both the AS group and surgery group were excellent. Although studies of larger numbers of patients are needed, at the present time, we can conclude that active surveillance can be used to manage HTTs that show no aggressive signs on imaging.

Ultrasonographic features of HTT have been demonstrated including round shape, hypoechogenicity, absence of calcifications, and the presence of peri- or intranodular vascularity [12-14]. Lee et al. showed that the appearance of HTT is often similar to that of follicular neoplasm [12]. Indeed, in our series, $85 \%$ of the patients who were preoperatively (cytologically) diagnosed as or highly suspected of having HTT and $79 \%$ of those who were pathologically diagnosed as having HTT showed a US class 3 (follicular tumor) (Tables 1 and 2). In addition, their intranodular and peripheral blood flows were generally high. Although it is impossible to diagnose HTT based only on these features, the features may contribute to the suspicion of HTT together with cytological findings. 
Table 2 Backgrounds and clinicopathological features of the patients diagnosed as having HTT based on pathological findings

\begin{tabular}{|c|c|c|c|c|}
\hline Variables & $\begin{array}{l}{ }^{1} \text { Diagnosed as or highly } \\
\text { suspected of HTT on } \\
\text { cytology and diagnosed as } \\
\text { HTT on pathology } \\
(n=22)\end{array}$ & $\begin{array}{l}{ }^{2} \text { Suspected of or } \\
\text { possible HTT on } \\
\text { cytology and diagnosed } \\
\text { as HTT on pathology } \\
(n=7)\end{array}$ & $\begin{array}{l}\text { Suspected of or } \\
\text { diagnosed as PTC on } \\
\text { cytology but diagnosed } \\
\text { as HTT on pathology } \\
\quad(n=5)\end{array}$ & $\begin{array}{c}\text { Total } \\
(n=34)\end{array}$ \\
\hline Median age at surgery, yrs & $59(38-75)$ & $57(38-72)$ & $49(46-71)$ & $57(38-75)$ \\
\hline Male & $2(9 \%)$ & $1(14 \%)$ & $1(20 \%)$ & $4(12 \%)$ \\
\hline Female & $20(91 \%)$ & $6(86 \%)$ & $4(80 \%)$ & $30(88 \%)$ \\
\hline \multicolumn{5}{|l|}{ US class: } \\
\hline Calcification only & 0 & 0 & $1(20 \%)$ & $1(3 \%)$ \\
\hline 2.5 & $1(5 \%)$ & 0 & 0 & $1(3 \%)$ \\
\hline 3 & $19(86 \%)$ & $7(100 \%)$ & $1(20 \%)$ & $27(79 \%)$ \\
\hline 3.5 & $2(9 \%)$ & 0 & 0 & $2(6 \%)$ \\
\hline 4 & 0 & 0 & $3(60 \%)$ & $3(9 \%)$ \\
\hline \multicolumn{5}{|l|}{ Intranodular blood flow: } \\
\hline Negative & 0 & 0 & $1(20 \%)$ & $1(3 \%)$ \\
\hline $1+$ & $6(27 \%)$ & $2(29 \%)$ & $1(20 \%)$ & $9(26 \%)$ \\
\hline $2+$ & $11(50 \%)$ & $5(71 \%)$ & $3(60 \%)$ & $19(56 \%)$ \\
\hline $3+$ & $5(23 \%)$ & 0 & 0 & $5(14 \%)$ \\
\hline \multicolumn{5}{|l|}{ Peripheral blood flow: } \\
\hline Negative & 0 & 0 & $1(20 \%)$ & $1(3 \%)$ \\
\hline $1+$ & $6(27 \%)$ & $1(14 \%)$ & 0 & $7(21 \%)$ \\
\hline $2+$ & $9(41 \%)$ & $4(57 \%)$ & $4(80 \%)$ & $17(50 \%)$ \\
\hline $3+$ & $7(32 \%)$ & $2(29 \%)$ & 0 & $9(26 \%)$ \\
\hline \multicolumn{5}{|l|}{$\begin{array}{l}\text { Membranous staining of MIB-1 on } \\
\text { cytological specimens: }\end{array}$} \\
\hline Yes & $5(83 \%)$ & 0 & 0 & $5(45 \%)$ \\
\hline No & $1(17 \%)$ & $4(100 \%)$ & $1(100 \%)$ & $6(55 \%)$ \\
\hline Not done & 16 & 3 & 4 & 23 \\
\hline \multicolumn{5}{|l|}{$\begin{array}{l}\text { Membranous staining of MIB-1 on } \\
\text { pathological specimens: }\end{array}$} \\
\hline Yes & $22(100 \%)$ & $7(100 \%)$ & $5(100 \%)$ & $34(100 \%)$ \\
\hline No & 0 & 0 & 0 & 0 \\
\hline Not done & 0 & 0 & 0 & 0 \\
\hline Median tumor size, $(\mathrm{mm})$ & $26(14-60)$ & $22(13-26)$ & $15(14-21)$ & $24(13-60)$ \\
\hline \multicolumn{5}{|l|}{ Extent of thyroidectomy: } \\
\hline Total & $3(14 \%)$ & 0 & $1(20 \%)$ & $4(12 \%)$ \\
\hline Subtotal or hemithyroidectomy & $19(86 \%)$ & $7(100 \%)$ & $4(80 \%)$ & $30(88 \%)$ \\
\hline \multicolumn{5}{|l|}{ Lymph node dissection: } \\
\hline $\mathrm{CND}+\mathrm{MND}$ & $1(5 \%)$ & 0 & 0 & $1(3 \%)$ \\
\hline CND & $8(36 \%)$ & $5(71 \%)$ & $5(100 \%)$ & $18(53 \%)$ \\
\hline Not done & $13(59 \%)$ & $2(29 \%)$ & 0 & $15(44 \%)$ \\
\hline $\begin{array}{l}\text { Median postoperative follow-up } \\
\text { months }\end{array}$ & $60(1-151)$ & $59(30-86)$ & $61(11-90)$ & $60(1-151)$ \\
\hline \multicolumn{5}{|l|}{ Recurrence of HTT: } \\
\hline Yes & 0 & 0 & 0 & 0 \\
\hline No & $22(100 \%)$ & $7(100 \%)$ & $5(100 \%)$ & $34(100 \%)$ \\
\hline
\end{tabular}

${ }^{1}$ Without the possibility of PTC on cytology. ${ }^{2}$ With the possibility of PTC on cytology. ${ }^{3}$ Tumors were pathologically diagnosed as PTC. HTT, hyalinizing trabecular tumor; PTC, papillary thyroid carcinoma; MND, modified radical neck dissection; CND, central node dissection. 
A cytological diagnosis is the most important factor to diagnose HTT, by discriminating it from PTC. However, the cytological features of HTT closely resemble those of PTC. Kuma et al. demonstrated that in cytologic specimens, intranuclear cytoplasmic inclusions were detected in $100 \%$ of HTTs and $75 \%$ of PTCs, and the incidences of nuclear grooves were $100 \%$ of the PTCs and $81.8 \%$ of the HTTs [6], which makes the differential diagnosis of these diseases difficult.

Hirokawa et al. demonstrated that membranous staining for MIB-1 is an important finding for diagnosing HTT [7, 8]. Indeed, all of the 34 surgical specimens in our present series were positive for membranous staining for MIB-1 (Table 2). However, MIB-1 immunostaining for cytological specimens was performed in only 11 patients, and only five of these (45\%) were positive (Table 2). This indicates that MIB-1 staining was much less stable in cytological specimens than surgical specimens. In addition, in our experience, it is often difficult to aspirate an adequate amount of tumor cells on cytology for MIB-1 immunostaining. Moreover, MIB-1 staining for HTT is not covered by health insurance in Japan. We have thus not routinely performed MIB-1 immunostaining for cytological specimens when other typical cytological findings of HTT could be detected. The indication of MIB-1 immunostaining for cytological specimens should be when HTT is suspected but its cytological evidence of HTT is not adequate.

Hino et al. reported that von Kossa staining (staining for calcification) is useful for discriminating HTT from PTC in surgical specimens [15]. In their series of 12 HTTs, all of the specimens were positive for tiny or small black dots in the basement of membranous stroma, whereas 25 of the 30 PTC specimens were negative for the dots. However, two (17\%) of the 12 HTTs and five (17\%) of the 30 PTCs showed a weak staining of tiny or small black dots, indicating that von Kossa staining could not perfectly discriminate between HTT and PTC.
In addition, this staining cannot be used for cytological specimens.

More recently, genetic studies indicated that the GLIS rearrangements, especially PAX8-GLIS3 gene fusion, are useful to diagnose HTT $[16,17]$. Nikiforova et al. reported that $P A X 8-G L I S 3$ gene fusion was identified in $13(93 \%)$ of 14 HTTs, and the remaining HTTs (7\%) showed PAX8-GLIS1 gene fusion [16]. Nikiforova et al. also noted that 220 PTCs revealed no PAX8-GLIS3 and one PTC showed PAX8-GLIS1 fusion. Marchio et al. demonstrated that all of 26 HTTs revealed PAX8-GLIS3 gene fusion, whereas no PAX8-GLIS3 could be detected in 237 control thyroid neoplasms. The analysis of the GLIS rearrangements should thus be considered promising for accurately diagnosing HTT, but it is difficult to make such an analysis in general hospitals.

This study has some limitations. It was a retrospective analysis, and we did not have any study protocols for patients who were diagnosed as or suspected of having HTT prospectively. Another limitation is that the diagnosis of HTT in the AS group was based only on cytology, indicating that there may be a possibility that the pathological diagnosis would differ from HTT if surgery was conducted, although the incidence should be low. Nevertheless, we were able to enroll a relatively large number of HTT patients and to determine the biological characteristics of HTTs when operated on and undergoing active/passive surveillance.

In summary, HTT can be diagnosed on cytology at a considerably high incidence, i.e., $65 \%$. If tumors have no possibility of being PTC based on cytology and do not show any aggressive signs such as significant extrathyroid extension and lymph node and/or distant metastasis, active surveillance could be accepted as one of the management strategies. If the possibility of PTC cannot be denied on cytology, immediate surgery is appropriate except for tumors measuring $\leq 1 \mathrm{~cm}$.

\section{References}

1. Carney JA, Ryan J, Goellner JR (1987) Hyalinizing trabecular adenoma of the thyroid gland. Am J Surg Pathol 11: $583-591$.

2. Goellner JR, Carney JA (1989) Cytologic features of fineneedle aspirates of hyalinizing trabecular adenoma of the thyroid. Am J Clin Pathol 91: 115-119.

3. Strong CJ, Garcia BM (1990) Fine needle aspiration cytology characteristics of hyalinizing trabecular adenoma of the thyroid. Acta Cytol 34: 359-362.

4. Akin MR, Nguyen GK (1999) Fine-needle aspiration biopsy cytology of hyalinizing trabecular adenomas of the thyroid. Diagn Cytopathol 20: 90-94.
5. Rothenberg HJ, Goellner JR, Carney JA (1999) Hyalinizing trabecular adenoma of the thyroid gland: recognition and characterization of its cytoplasmic yellow body. Am J Surg Pathol 23: 118-125.

6. Kuma S, Hirokawa M, Miyauchi A, Kakudo K, Katayama S (2003) Cytologic features of hyalinizing trabecular adenoma of the thyroid. Acta Cytol 47: 399-404.

7. Hirokawa $M$, Shimizu $M$, Manabe $T$, Kuroda $M$, Mizoguchi Y (1995) Hyalinizing trabecular adenoma of the thyroid: its unusual cytoplasmic immunopositivity for MIB1. Pathol Int 45: 399-401.

8. Hirokawa M, Carney JA (2000) Cell membrane and 
cytoplasmic staining for MIB-1 in hyalinizing trabecular adenoma of the thyroid gland. Am J Surg Pathol 24: 575578.

9. Carney JA, Hirokawa M, Lloyd RV, Papotti M, Sebo TJ (2008) Hyalinizing trabecular tumors of the thyroid gland are almost all benign. Am J Surg Pathol 32: 1877-1889.

10. Takada N, Hirokawa M, Ohbayashi C, Nishikawa T, Itoh $\mathrm{T}$, et al. (2018) Re-evaluation of MIB-1 immunostaining for diagnosing hyalinizing trabecular tumour of the thyroid: Semi-automated techniques with manual antigen retrieval are more accurate than fully automated techniques. Endocr J 65: 239-244.

11. Yokozawa T, Fukata S, Kuma K, Matsuzuka F, Kobayashi A, et al. (1996) Thyroid cancer detected by ultrasoundguided fine-needle aspiration biopsy. World J Surg 20: 848-853.

12. Lee S, Han BK, Ko EY, Oh YL, Choe JH, et al. (2011) The ultrasonography features of hyalinizing trabecular tumor of the thyroid are more consistent with its benign behavior than cytology or frozen section readings. Thyroid 21: 253-259.

13. Choi WL, Baek JH, Ha EJ, Choi YJ, Hong MJ, et al.
(2015) The ultrasonography features of hyalinizing trabecular tumor of the thyroid gland and the role of fine needle aspiration cytology and core needle biopsy in its diagnosis. Acta Radiol 56: 1113-1118.

14. Jang H, Park CK, Son EJ, Kim EK, Kwak JY, et al. (2016) Hyalinizing trabecular tumor of the thyroid: diagnosis of a rare tumor using ultrasonography, cytology, and intraoperative frozen sections. Ultrasonography 35: 131139.

15. Hino R, Motoi N, Toda $\mathrm{K}$, Ebina A, Yamada $\mathrm{K}$, et al. (2018) Stromal tiny black dots, like "sugar-coated", of von Kossa stain is a diagnostic clue to hyalinizing trabecular tumor of the thyroid gland. Pathol Int 68: 176-182.

16. Nikiforova MN, Nikitski AV, Panebianco F, Kaya C, Yip L, et al. (2019) GLIS rearrangement is a genomic hallmark of hyalinizing trabecular tumor of the thyroid gland. Thyroid 29: 161-173.

17. Marchio C, Da Cruz Paula A, Gularte-Merida R, Basill T, Brandes A, et al. (2019) PAX-GLIS3 gene fusion is a pathognomonic genetic alteration of hyalinizing trabecular tumors of the thyroid. Mod Pathol 32: 1734-1743. 